\title{
Lactobacillus acidophilus Improves Intestinal Inflammation in an Acute Colitis Mouse Model by Regulation of Th17 and Treg Cell Balance and Fibrosis Development
}

\author{
Jin-Sil Park, ${ }^{1, *}$ Jeong Won Choi, ${ }^{1, *}$ Joo Yeon Jhun, Ji Ye Kwon, ${ }^{1}$ Bo-In Lee, \\ Chul Woo Yang, Sung-Hwan Park, ${ }^{1,4}$ and Mi-La Cho ${ }^{1}$ \\ ${ }^{1}$ The Rheumatism Research Center, Catholic Research Institute of Medical Science, College of Medicine; \\ ${ }^{2}$ Division of Gastroenterology, Department of Internal Medicine, Seoul St. Mary's Hospital, College of Medicine; \\ ${ }^{3}$ Division of Nephrology, Department of Internal Medicine, Seoul St. Mary's Hospital, College of Medicine; \\ ${ }^{4}$ Divison of Rheumatology, Department of Internal Medicine, Seoul St. Mary's Hospital, College of Medicine; \\ The Catholic University of Korea, Seoul, Korea.
}

\begin{abstract}
Disruption of the balance among the microbiota, epithelial cells, and resident immune cells in the intestine is involved in the pathogenesis of inflammatory bowel disease (IBD). Probiotics exert protective effects against IBD, and probiotic commensal Lactobacillus species are common inhabitants of the natural microbiota, especially in the gut. To investigate the effects of Lactobacillus acidophilus on the development of IBD, L. acidophilus was administered orally in mice with dextran sodium sulfate (DSS)-induced colitis. DSS-induced damage and the therapeutic effect of L. acidophilus were investigated. Treatment with $L$. acidophilus attenuated the severity of DSS-induced colitis. Specifically, it suppressed proinflammatory cytokines such as interleukin (IL)-6, tumor necrosis factor- $\alpha$, IL- $1 \beta$, and IL-17 in the colon tissues, which are produced by T helper (Th) 17 cells. Moreover, in vitro L. acidophilus treatment directly induced T regulatory (Treg) cells and the production of IL-10, whereas the production of IL-17 was suppressed in splenocytes. In addition, we found that $L$. acidophilus treatment decreased the levels of $\alpha$-smooth muscle actin, a marker of activated myofibroblasts, and type I collagen compared with control mice. These results suggest that $L$. acidophilus may be a novel treatment for IBD by modulating the balance between Th17 and Treg cells, as well as fibrosis development.
\end{abstract}

KEYWORDS: $\bullet$ fibrosis $\bullet$ inflammatory bowel disease $\bullet$ L. acidophilus $\bullet$ Th17 cell $\bullet$ Treg cell

\section{INTRODUCTION}

I NFLAMMATORY Bowel DiSEASE (IBD) comprises Crohn's disease (CD) and ulcerative colitis (UC), and is characterized by chronic inflammatory relapse of the gastrointestinal tract caused by multiple genetic and environmental factors. ${ }^{1}$ IBD arises from an uncontrolled immune response to resident antigens and the microbiota, resulting in complications such as perforating ulcers and fibrosis. ${ }^{2-4}$ Intestinal fibrosis is a major complication of IBD. Uncontrolled production and accumulation of excess extracellular matrix components result in dysfunctional wound healing and collagen accumulation, and induce excessive fibrosis and possible intestinal obstruction. ${ }^{5,6}$

*Jin-Sil Park and Jeong Won Choi contributed equally to this work.

Manuscript received 26 April 2017. Revision accepted 12 November 2017.

Address correspondence to: Mi-La Cho, PhD, Rheumatism Research Center, Catholic Institutes of Medical Science, College of Medicine, The Catholic University of Korea, 222, Banpo-daero, Seocho-gu, Seoul 06591, Republic of Korea, E-mail: iammila@ catholic.ac.kr
In states of intestinal homeostasis, disruption in the balance among the microbiota, intestinal epithelial cells, and resident immune cells is involved in the pathogenesis of IBD. ${ }^{7}$ Numerous studies have suggested that $T$ cells reactive to microbiota antigens are vital in regulating intestinal homeostasis. ${ }^{8}$ For many years, IBD was thought to be associated with interferon- $\gamma$-producing $\mathrm{T}$ helper $(\mathrm{Th}) 1$ cells in $\mathrm{CD}$ and Th2 cells in UC. However, more recent studies have suggested that an abnormal mucosal immune response is associated with interleukin (IL)-17-producing T (Th17) cells., ${ }^{9,10}$ For instance, IL-17 expression, the number of Th17 cells, and serum IL-17 levels were increased in UV and CD patients. ${ }^{1-13}$ IL-17A-deficient and IL-17 receptor-knockout mice were protected against colonic inflammation in dextran sodium sulfate (DSS)-induced colitis and trinitrobenzenesulfonic acid-induced colitis models, respectively. ${ }^{14,15}$

Forkhead box P3 (Foxp3)-expressing T regulatory (Treg) cells play a critical role in maintaining immune homeostasis and controlling excessive inflammatory responses in the innate and adaptive immune systems. Accumulating scientific evidence has revealed that Treg cells play an essential role in the pathogenesis of autoimmune diseases as well as 
IBD. ${ }^{16}$ Treg cells that secrete IL-10, an anti-inflammatory cytokine, are important for the maintenance of intestinal homeostasis and induce spontaneous colitis in IL-10deficient mice. ${ }^{17}$

Several studies have demonstrated that probiotics have various advantages; thus, supplementing with probiotics may be a promising alternative therapy for IBD. ${ }^{18,19}$ However, the mechanism underlying the protective effects exerted by probiotics in IBD is still largely unknown. Commensal Lactobacillus species are predominant in the microflora of the gut. These species restore homeostasis in intestinal disorders and thus play a protective role against IBD. ${ }^{20}$ Lactobacillus acidophilus is commonly used as a probiotic.

This study sought to determine the involvement of $L$. acidophilus in the modulation of DSS-induced colitis by restoration of the balance of inflammatory cytokines and Th17/Treg cells. In particular, the effects of $L$. acidophilus on intestinal fibrosis in IBD were investigated. In this study, L. acidophilus exerted simultaneous regulation of Th17 and Treg cells in a DSS-induced colitis model, both in vitro and in vivo. Furthermore, intestinal fibrosis was reduced by $L$. acidophilus treatment in the DSS-induced colitis model.

\section{MATERIALS AND METHODS}

Mice

Eight-week-old male C57BL/6 mice were purchased from Orient Bio, Inc. (Seongnam, Korea). The animals were maintained under specific pathogen-free conditions at the Institute of Medical Science of the Catholic University of Korea and were fed standard mouse chow and water. All experimental procedures were approved by the Department of Laboratory Animals, Institutional Animal Care and Use Committee (IACUC) of the School of Medicine, the Catholic University of Korea, and conformed with all National Institutes of Health (USA) guidelines (Permit number: 2016-0244-01).

\section{Induction of colitis and $\mathrm{L}$. acidophilus administration}

C57BL/6 mice were administered 3\% DSS (MP Biomedicals, Santa Ana, CA, USA) in their drinking water for 4 days. Mice were randomly assigned to three groups $(n=3 /$ group) as follows: Group 1 mice were administered normal drinking tap water ( $n=3$ /group), Group 2 mice were administered DSS in drinking tap water ( $n=3 /$ group), and Group 3 mice were administered L. acidophilus $\left(8 \times 10^{10}\right.$ CFU/kg) 3 days after DSS administration. Body weight and disease activity index (DAI) scores were monitored daily. L. acidophilus $\left(8 \times 10^{10} \mathrm{CFU} / \mathrm{kg}\right)$ was administered. Data are representative of four independent experiments with reproducible results.

\section{Assessment of inflammation}

During the experimental period, the severity of colitis was assessed daily by measuring percentage body weight change and DAI. The DAI was calculated as described previously. ${ }^{21}$ Body weight loss (score: 0, none; 1, 0-5\%; 2, 6-10\%; 3, 11-
$15 \% ; 4,16-20 \% ; 5,21-25 \%$; and $6,26-30 \%$ ), stool consistency (score: 0 , normal stools; 1 , soft stools; and 2, liquid stools), and rectal bleeding (score: 0 , negative fecal occult blood; 1, positive fecal occult blood; and 2, visible rectal bleeding) were assessed daily in each mouse.

\section{Histopathological analysis}

Colon tissue sections ( $5 \mu \mathrm{m}$ thick) were fixed and embedded in $10 \%(\mathrm{v} / \mathrm{v})$ neutral buffered formalin (SigmaAldrich, St. Louis, MO) and stained with hematoxylin and eosin. Loss of epithelium, crypt damage, depletion of goblet cells, and infiltration of inflammatory cells were assessed in these sections. Histological evaluation and scoring were performed for the loss of epithelium ( 0 , no loss of epithelium; $1,0-5 \%$ loss of epithelium; 2, 5-10\% loss of epithelium; and $4,>10 \%$ loss of epithelium), crypt damage ( 0 , no damage; 1 , 0-10\% loss of crypt; 2, 10-20\% loss of crypt; and 3,>20\% loss of crypt), depletion of goblet cells ( 0 , none; 1 , mild; 2 , moderate; and 3 , severe), and infiltration of inflammatory cells $(0$, none; 1 , mild; 2 , moderate; and 3 , severe). The total score was calculated as the sum of each of the above scores.

\section{Immunohistochemistry}

Sections were treated with $3 \%(\mathrm{v} / \mathrm{v}) \mathrm{H}_{2} \mathrm{O}_{2}$ in methanol to block the endogenous peroxidase activity. Immunohistochemistry was performed using the Envision Detection ${ }^{\mathrm{TM}}$ kit (DAKO Agilent Technologies, Inc., Santa Clara, CA, USA). Tissue sections were incubated with primary antibodies against IL-10, Foxp3 (Santa Cruz Biotechnology, Santa Cruz, CA, USA), tumor necrosis factor alpha (TNF- $\alpha$ ), IL$1 \beta$, IL-17, IL-6, $\alpha$-smooth muscle actin ( $\alpha$-SMA), and type I collagen (Col-I) (Abcam) for $2 \mathrm{~h}$ at room temperature. Sections were then incubated with a biotinylated secondary antibody and streptavidin-peroxidase complex for $30 \mathrm{~min}$. The final colored products were developed using chromogen diaminobenzidine and the sections were examined under a photomicroscope (Olympus, Tokyo, Japan). Positive cells were enumerated visually by four individuals, and the mean values were calculated.

\section{Isolation and stimulation of splenocytes}

Mouse spleens were sieved through a $40-\mu$ m nylon cell strainer (BD Falcon), and red blood cells were lysed using hypotonic ammonium-chloride-potassium buffer $(0.15 \mathrm{mM}$ $\mathrm{NH}_{4} \mathrm{Cl}, 1 \mathrm{mM} \mathrm{KCO}$, and $0.1 \mathrm{mM}$ EDTA, $\mathrm{pH}$ 7.4). The remaining splenocytes were maintained in RPMI 1640 medium containing 5\% fetal bovine serum. Cells were cultured in the presence of plate-bound anti-CD3 $(0.5 \mu \mathrm{g} / \mathrm{mL})$ and soluble anti-CD28 $(1 \mu \mathrm{g} / \mathrm{mL})$ with or without $L$. acidophilus for 3 days. HT-29 cell lines were stimulated with $L$. acidophilus in the presence of lipopolysaccharide (LPS; $100 \mathrm{ng}$ / $\mathrm{mL}$ ) for 3 days.

\section{Real-time polymerase chain reaction}

The LightCycler 2.0 instrument (Roche Diagnostics, software version 4.0) was used for polymerase chain 
reaction $(\mathrm{PCR})$ amplification. All reactions were performed using LightCycler FastStart DNA Master SYBR Green I (Takara) according to the manufacturer's instructions. The following primers were used: IL-6, 5'-AGA CAG CCA CTC ACC TCT TCA G-3' (sense) and 5'-TTC TGC CAG TGC CTC TTT GCT G-3' (antisense); TNF- $\alpha, 5^{\prime}$-GCC TCT TCT CCT TCC TGA TCG T- $3^{\prime}$ (sense) and $5^{\prime}$-CTC GGC AAA GTC GAG ATA GTC G-3' (antisense); IL-1 $\beta$, 5'-GGA CAA GCT GAG GAA GAT GC-3' (sense) and 5'-TCG TTA TCC CAT GTG TCG AA-3' (antisense); and $\beta$-actin, $5^{\prime}$ GGA CTT CGA GCA AGA GAT GG-3' (sense) and $5^{\prime}$ TGT GTT GGG GTA CAG GTC TTT G-3' (antisense). The mRNA levels were normalized to that of $\beta$-actin.

\section{Enzyme-linked immunosorbent assay}

The levels of IL-10 and IL-17A in the supernatants of murine splenocyte cultures were measured using sandwich enzyme-linked immunosorbent assays (ELISAs; R\&D Systems). Horseradish peroxidase-avidin (R\&D Systems) was used for color development. Absorbance was measured at $405 \mathrm{~nm}$ on an ELISA microplate reader (Molecular Devices, Sunnyvale, CA, USA).

\section{Flow cytometry}

Splenocytes were immunostained with various combinations of the fluorescence-conjugated antibodies CD4-PerCP (eBioscience, San Diego, CA, USA) and CD25-APC (Biolegend, San Diego, CA, USA). These cells were also stained intracellularly with an antibody against Foxp3-PE (eBioscience). Before intracellular staining, cells were stimulated with $25 \mathrm{ng} /$ $\mathrm{mL}$ phorbol myristate acetate (Sigma-Aldrich) and $250 \mathrm{ng} / \mathrm{mL}$ ionomycin for $4 \mathrm{~h}$. Intracellular staining was conducted using an intracellular staining kit (eBioscience) according to the manufacturer's protocol. Flow cytometry was performed using a FACS Calibur (BD Biosciences, San Diego, CA, USA), and the data were analyzed using Flow Jo software, version 7.6 (TreeStar, Ashland, OR, USA).

\section{Statistical analysis}

All statistical analyses were performed using GraphPad Prism (version 4 for Windows; GraphPad Software). Normally distributed continuous data were analyzed using the parametric Student's $t$ test. Differences in mean values among the various groups were analyzed by ANOVA followed by Bonferroni post hoc test. Experimental values are presented as mean \pm standard deviation. A value of $P<.05$ (two-tailed) was considered to indicate statistical significance.

\section{RESULTS}

\section{L. acidophilus attenuates DSS-induced colitis severity}

To determine whether L. acidophilus can modulate the development and severity of colitis in vivo, L. acidophilus was administered to mice with DSS-induced colitis. The length of the colon was significantly decreased in mice with DSS-induced colitis compared with normal mice. Treatment with L. acidophilus effectively prevented this decrease in colon length (Fig. 1A). Microscopic examination of the colon demonstrated that administration of L. acidophilus resulted in mucosal architecture recovery compared with vehicle-treated mice. Moreover, the histological scores of the L. acidophilus-treated group were decreased (Fig. 1B). We evaluated DSS-induced damage and the protective effect of L. acidophilus by measuring body weight. Body weight gradually increased in the normal group. Mice in the vehicle-treated DSS-induced colitis group maintained their body weight during the first 6 days, but then displayed significant weight loss from day 8 onward as a result of severe colitis. However, this reduction was less in L. acidophilustreated mice (Fig. 1C). Symptoms of colitis, including weight loss, changes in stool consistency, and bleeding, were evaluated separately as described previously. ${ }^{22}$ The highest DAI score was observed in the vehicle-treated DSS-induced colitis group, while DAI scores were decreased in the L. acidophilus-treated group (Fig. 1D).

\section{L. acidophilus inhibits inflammatory cytokine expression in DSS-induced colitis}

The protective effects of $L$. acidophilus against colitis as mediated by negative regulation of inflammatory mediators were assessed by immunostaining for inflammatory cytokines in colon sections (Fig. 2A). Vehicle-treated mice with DSS-induced colitis exhibited higher levels of IL- 6 , TNF- $\alpha$, and IL- $1 \beta$ in colon tissue. In contrast, the levels of these inflammatory mediators were lower in the colon tissue of the L. acidophilus-treated group compared with the vehicletreated group. The HT-29 cell line, a human colorectal adenocarcinoma cell line, was treated with L. acidophilus in the presence of LPS. The expression levels of $I L-6, T N F-\alpha$, and $I L-1 \beta$ were subsequently determined by real-time PCR (Fig. 2B). In vitro treatment with L. acidophilus decreased the levels of $I L-6, T N F-\alpha$, and $I L-1 \beta$.

L. acidophilus inhibits the level of $I L-17$, but induces the level of Foxp3 and IL-10 in DSS-induced colitis

The expression level of IL-17, which is mainly produced by Th17 cells in colon tissue, of the vehicle-treated and $L$. acidophilus-treated DSS-induced colitis groups was evaluated by immunohistochemistry. As shown in Figure 3, mice treated with L. acidophilus showed decreased level of IL-17. In contrast, the level of Foxp3, a master regulator of Treg cells $^{23}$ and IL-10 production, was increased in the colon tissue of the L. acidophilus-treated DSS-induced colitis model compared with the vehicle-treated group.

\section{L. acidophilus promotes Treg differentiation among murine splenocytes}

To determine the effects of $L$. acidophilus on Treg cell differentiation in vitro, murine splenocytes were cultured in the presence of anti-CD3 and anti-CD28 antibodies with or without L. acidophilus for $72 \mathrm{~h}$. After stimulation, in vitro 
A

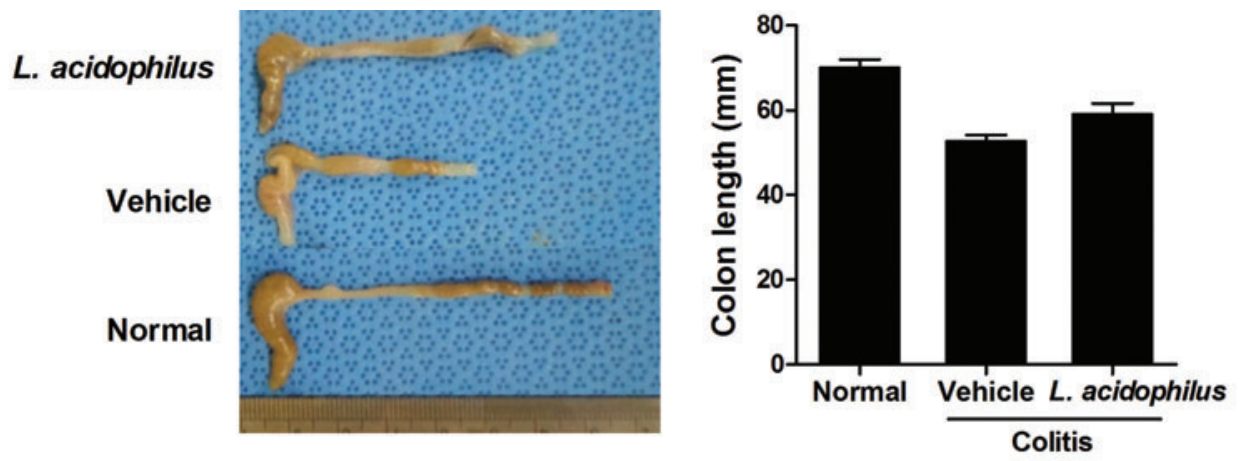

B
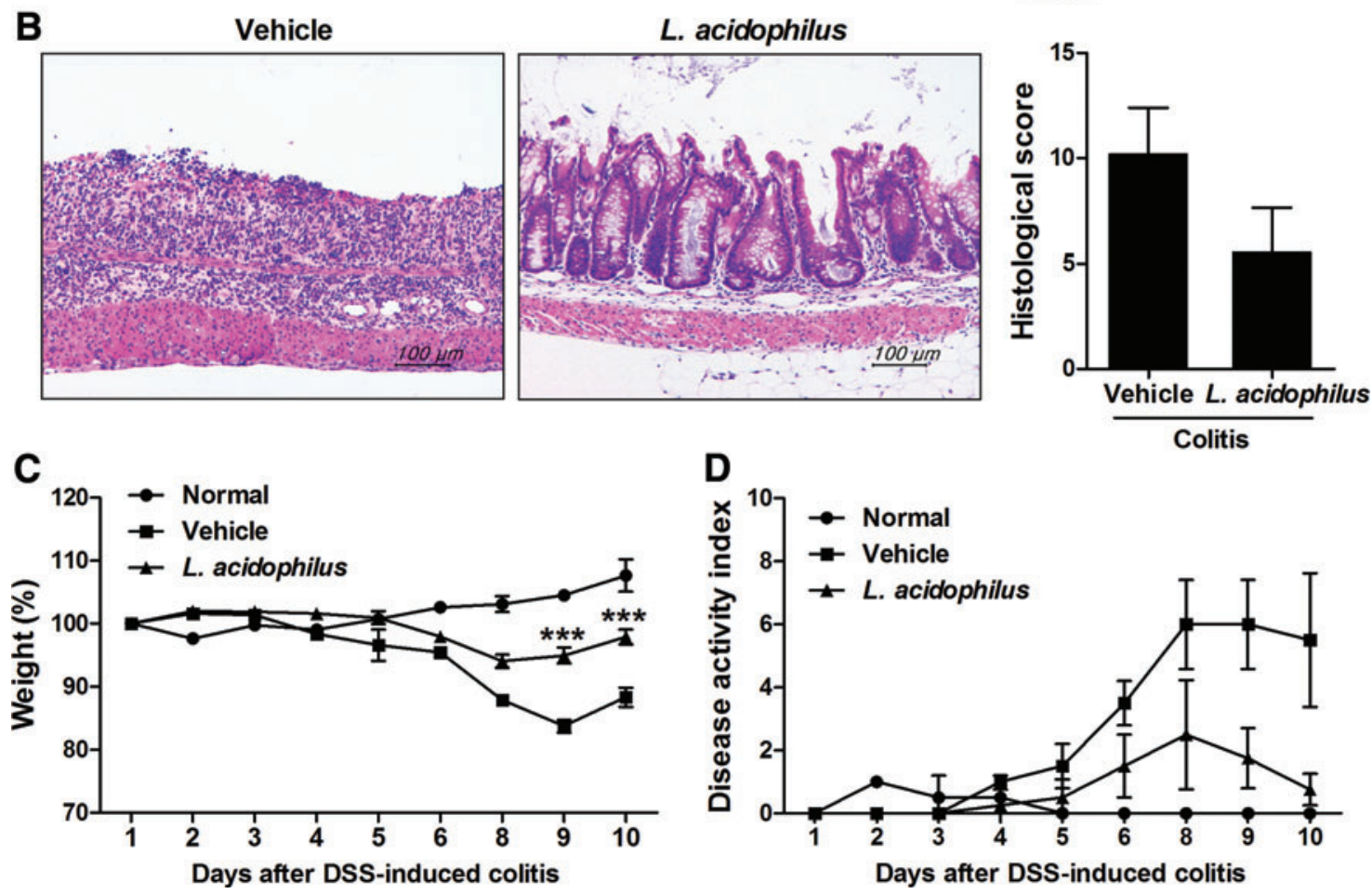

FIG. 1. Lactobacillus acidophilus attenuated the clinical symptoms of DSS-induced colitis. C57BL/6 mice were treated orally with $3 \%$ DSS in distilled water ad libitum from day 0 to day 4 . Thereafter, mice received regular drinking water. On the third day after DSS administration, $L$. acidophilus $\left(8 \times 10^{10} \mathrm{CFU} / \mathrm{kg}\right.$ ) was administered orally ( $n=3$ /group). (A) Assessment of the colon length of mice with DSS-induced chronic colitis on day 10. Macroscopic images of the colon are shown (left panel). The colon length of each mouse was measured (right panel). (B) Representative hematoxylin and eosin staining of the colon on day 10 . Scale bar $=100 \mu \mathrm{m}$. (C) Changes in body weight were measured. Body weight was presented as the percentage of the initial weight on day 0. (D) The DAI was monitored daily, excluding day 7 . Data are presented as mean \pm SD from four independent experiments. $* * * P<.001$ determined using a two-way ANOVA with Bonferroni post-test. Vehicle versus $L$. acidophilus treatment. DAI, disease activity index; DSS, dextran sodium sulfate; SD, standard deviation. Color images available online at www.liebertpub.com/jmf

treatment with $L$. acidophilus increased the proportion of $\mathrm{CD} 4^{+} \mathrm{CD} 25^{+} \mathrm{Foxp}^{+}$Treg cells, as assessed by flow cytometry (Fig. 4A). Spontaneous production of IL-17 and IL-10 by cultured splenocytes was measured by ELISA. Treatment with $L$. acidophilus induced the production of IL-10, whereas it suppressed the production of IL-17 (Fig. 4B).

\section{L. acidophilus reduces colitis-associated intestinal fibrosis and oxidative stress}

To investigate the antifibrotic effects of L. acidophilus on DSS-induced colitis-associated fibrosis, colon sections were stained with Masson's trichrome (Fig. 5A). Oral administration of L. acidophilus inhibited the intensity and area of blue staining in the colonic muscular layers compared with the vehicle-treated group. Transdifferentiation of fibroblasts toward myofibroblasts is a critical step in the fibrogenic process of chronic colitis. To evaluate the effect of L. acidophilus on the localization of myofibroblasts in the mucosa and submucosa, immunohistochemistry was performed for $\alpha$-SMA, a marker of activated myofibroblasts, in mice with DSS-induced colitis on day 10 . The number of $\alpha-\mathrm{SMA}^{+}$myofibroblasts was significantly decreased in the colonic mucosa of the L. acidophilus-treated DSS-induced 
A
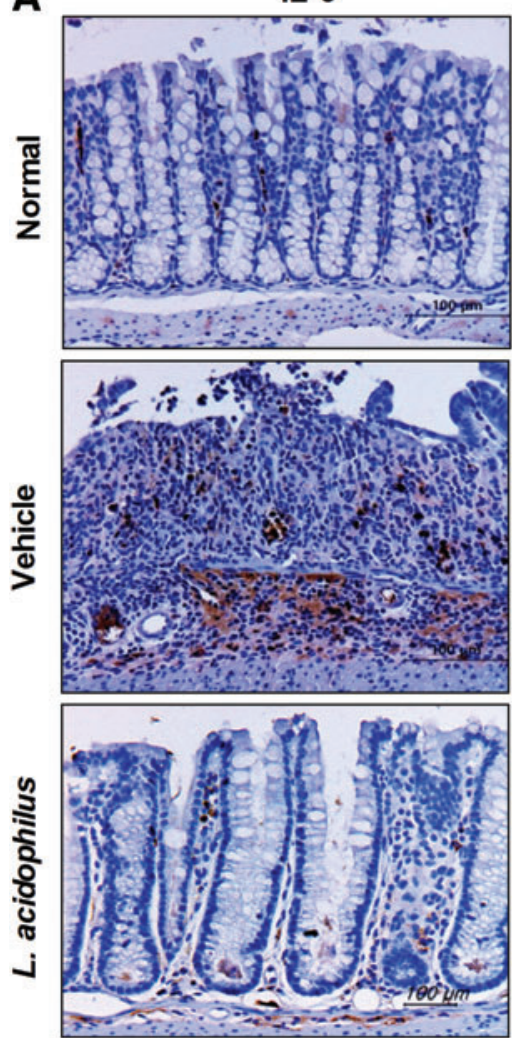

TNF- $\alpha$
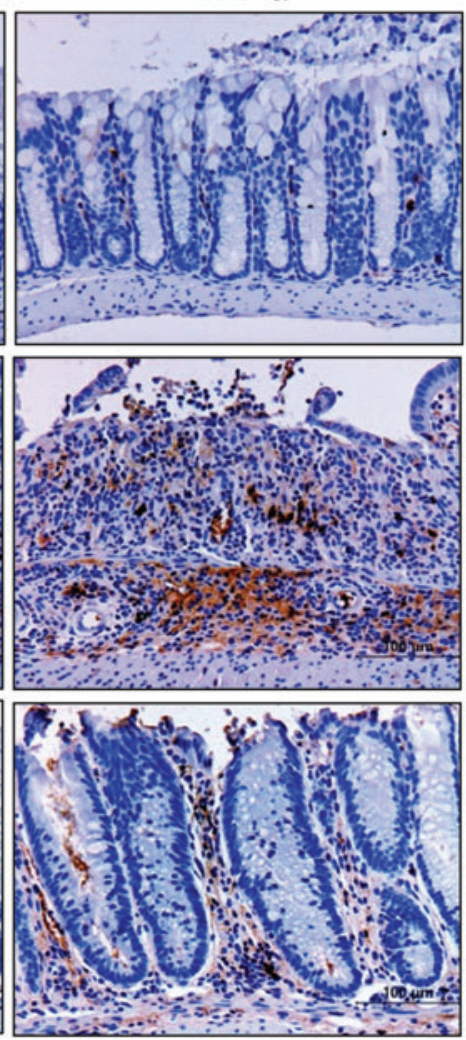

IL-1 $\beta$
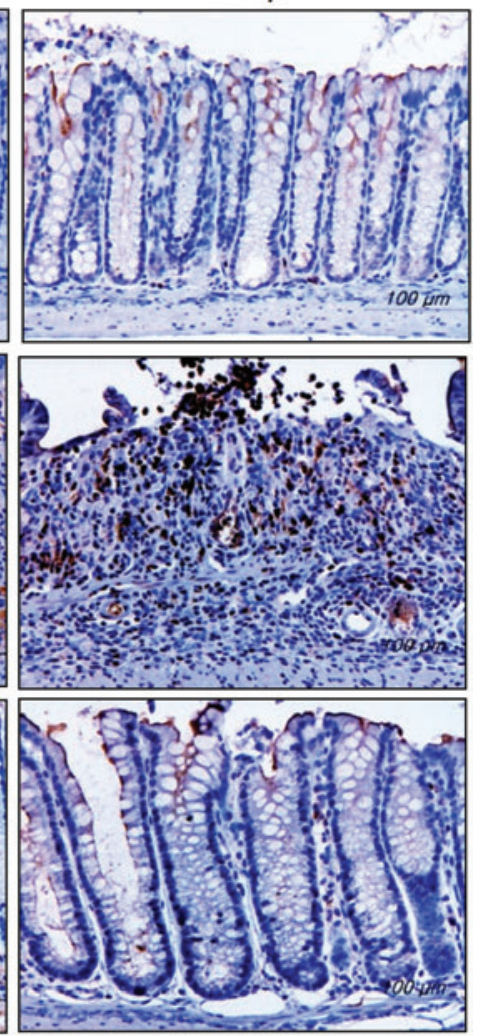
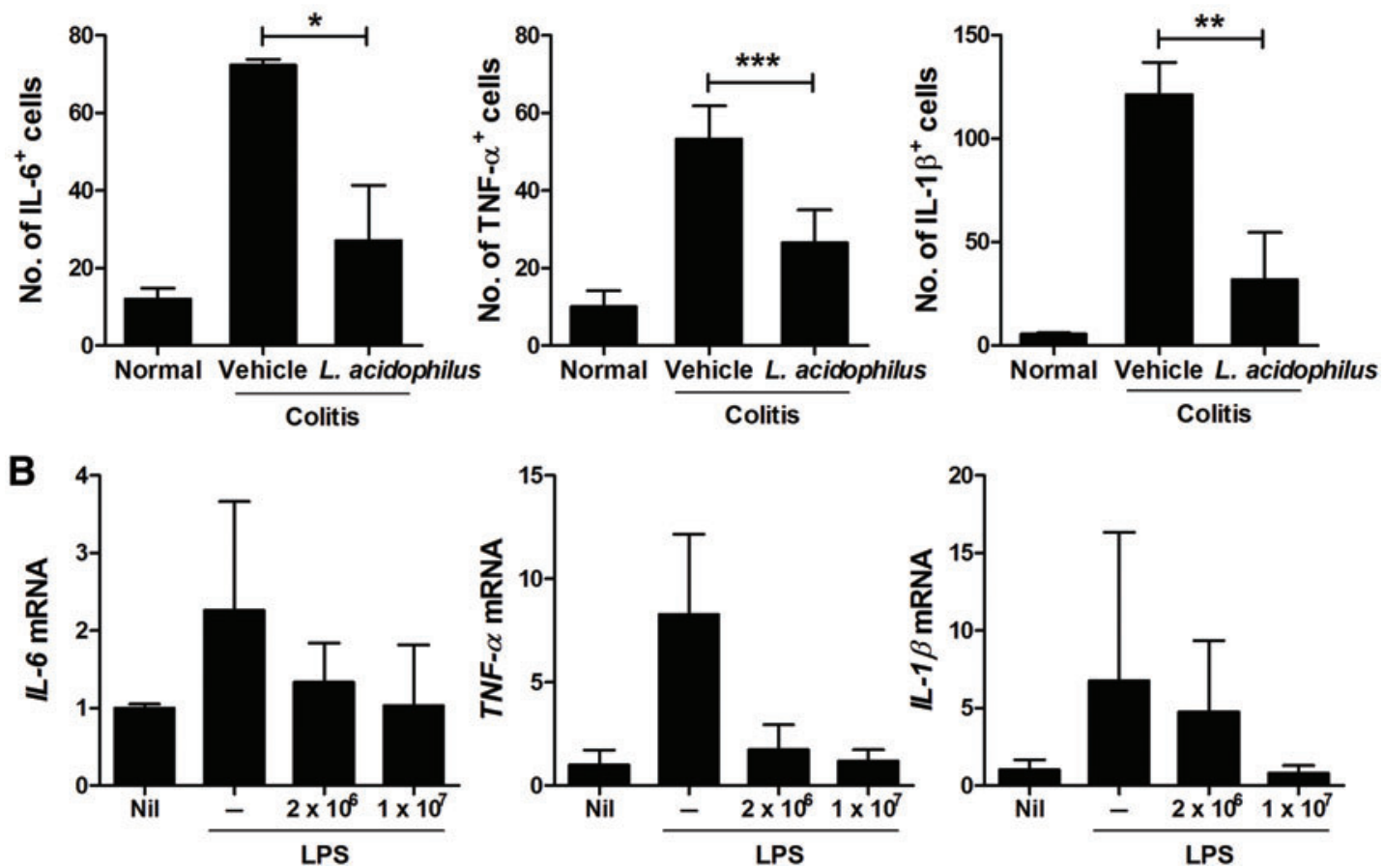

FIG. 2. L. acidophilus inhibits inflammatory cytokines in DSS-induced colitis. C57BL/6 mice were treated orally with $3 \%$ DSS in distilled water ad libitum from day 0 to 5. Thereafter, mice received regular drinking water. On day 3 after DSS administration, L. acidophilus $\left(8 \times 10^{10}\right.$ CFU $/ \mathrm{kg}$ ) was administered orally ( $n=3 /$ group). (A) On the 10th day after DSS administration, tissue sections were obtained from the colon and stained with antibodies against IL-6, TNF- $\alpha$, and IL- $1 \beta$. The pictures depict representative histological features, and the results are presented as the mean \pm SD number of antibody-positive cells ( $n=3$ /group). Scale bar $=100 \mu \mathrm{m}$. (B) HT-29 cells were treated with $L$. acidophilus $\left(2 \times 10^{6}\right.$ CFU or $\left.1 \times 10^{7} \mathrm{CFU} / \mathrm{mL}\right)$ in the presence of LPS $(100 \mathrm{ng} / \mathrm{mL})$ for $48 \mathrm{~h}$. mRNA expression of $I L-6, T N F-\alpha$, and $I L-1 \beta$ was analyzed by real-time PCR. $* P<.05, * * P<.01, * * * P<.001$ determined using a Student's $t$ test. Vehicle $v s$. L. acidophilus treatment. IL, interleukin; LPS, lipopolysaccharide; PCR, polymerase chain reaction; TNF- $\alpha$, tumor necrosis factor alpha. Color images available online at www.liebertpub.com/jmf 

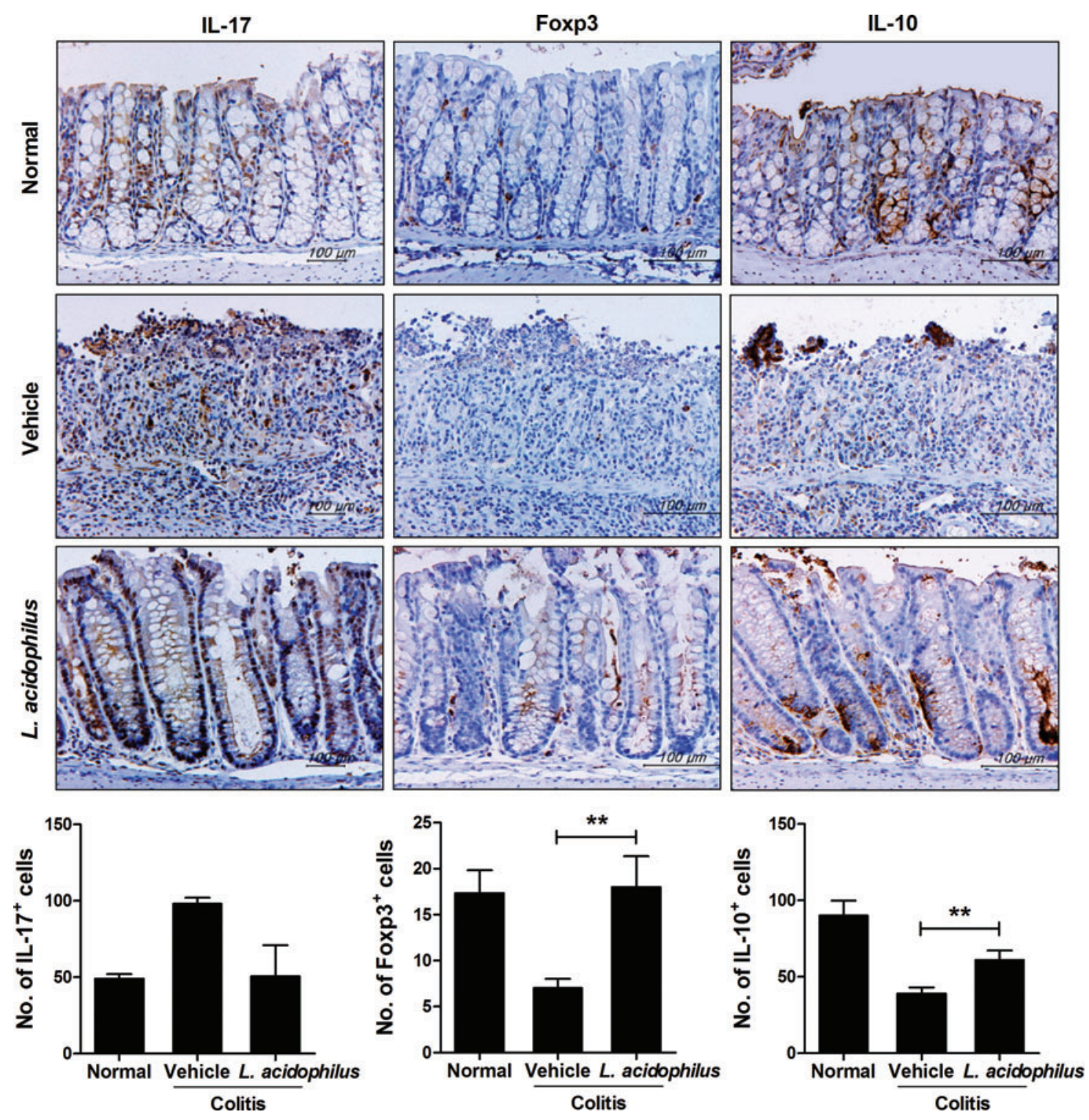

FIG. 3. L. acidophilus modulates the expression of IL-17 and Foxp3 in DSS-induced colitis. C57BL/6 mice were treated orally with 3\% DSS in distilled water ad libitum from day 0 to 5 . Thereafter, mice received regular drinking water. On the third day after DSS administration, $L$. acidophilus $\left(8 \times 10^{10} \mathrm{CFU} / \mathrm{kg}\right.$ ) was administered orally ( $n=3$ /group). On the 10th day after DSS administration, tissue sections were obtained from the colon and stained with antibodies against IL-17, Foxp3, and IL-10. The pictures depict representative histological features, and the results are presented as the mean \pm SD number of antibody-positive cells $(n=3 /$ group). Scale bar $=100 \mu \mathrm{m}$. $* * P<.01$ determined using a Student's $t$ test. Vehicle versus L. acidophilus treatment. Foxp3, forkhead box P3. Color images available online at www.liebertpub.com/jmf

colitis group compared with the vehicle-treated group (Fig. 5B). Increased deposition of type I collagen plays a major role in fibrosis in intestinal stricture formation. The number of Col- $\mathrm{I}^{+}$cells was also significantly decreased in the colonic mucosa of mice with $L$. acidophilus-treated DSS-induced colitis compared with vehicle-treated mice (Fig. 5C). To assess the effects of L. acidophilus on apoptosis and oxidative stress, immunohistochemistry was performed for TUNEL and 8-OHG, a marker of oxidative stress, in the colon. The numbers of $8-\mathrm{OHG}^{+}$cells and TUNEL $^{+}$cells were significantly increased in the colonic mucosa of vehicle-treated mice with DSS-induced colitis, but decreased in the colonic mucosa of L. acidophilustreated mice with DSS-induced colitis (Fig. 6).

\section{DISCUSSION}

The aim of this study was to elucidate the role of $L$. acidophilus, the most commonly used probiotic, in a DSSinduced colitis mouse model. Treatment with $L$. acidophilus prevented weight loss and shortening of the colon in these mice. The DAI and histological changes were reduced in the 


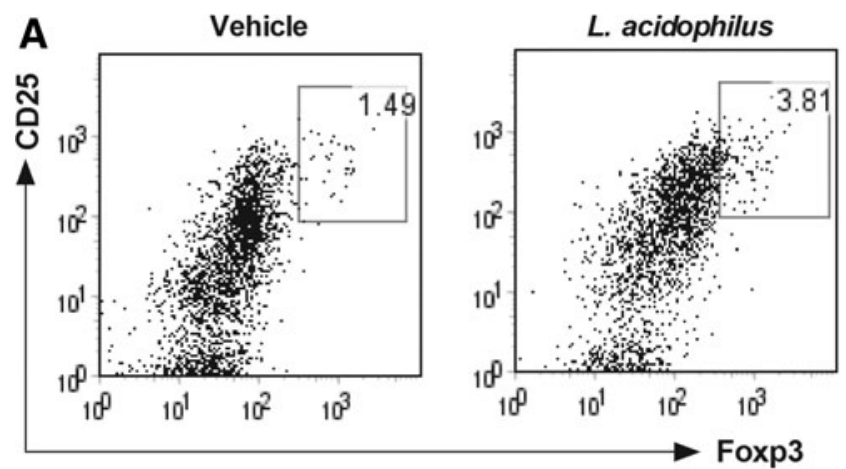

B

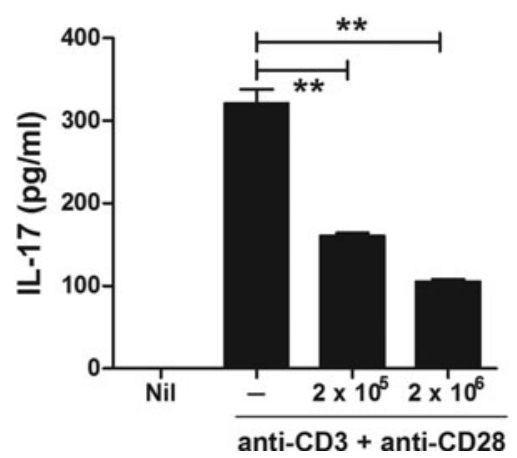

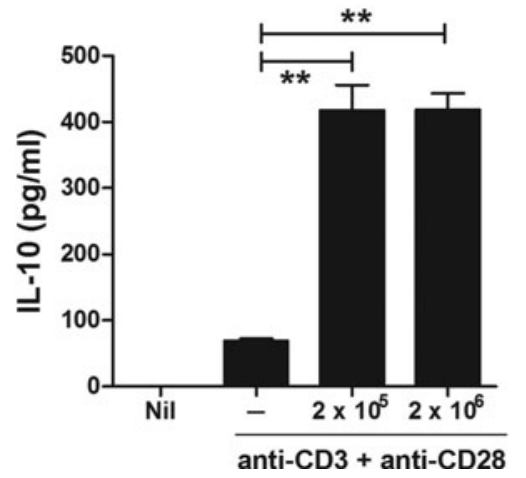

FIG. 4. L. acidophilus promotes Treg differentiation among murine splenocytes. Splenocytes were isolated from the spleens of C57BL/6 mice and stimulated with anti-CD3 and antiCD28 antibodies with or without $L$. acidophilus $\left(2 \times 10^{5} \mathrm{CFU}\right.$ or $2 \times 10^{6}$ $\mathrm{CFU} / \mathrm{mL}$ ) for 3 days. (A) After 3 days, the cells were stimulated with phorbol myristate acetate and ionomycin for $4 \mathrm{~h}$ and then subjected to staining with antibodies against CD4, CD25, and Foxp3 for flow cytometric analysis. Graphs represent mean \pm SD. (B) IL-17 and IL-10 levels in cell culture supernatants were measured by ELISA. $* * P<.01$ determined using a Student's $t$ test. Vehicle versus L. acidophilus treatment. CD, Crohn's disease; ELISA, enzyme-linked immunosorbent assay; Treg, $\mathrm{T}$ regulatory.

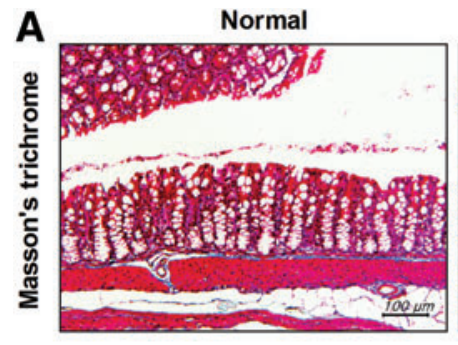

B

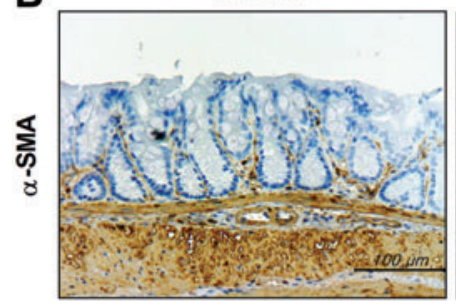

C

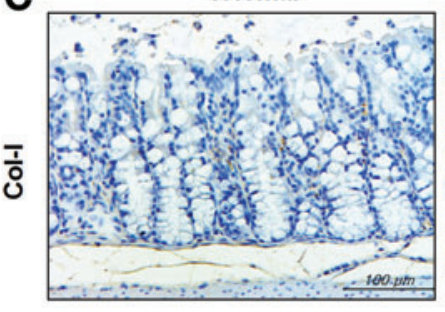

Vehicle

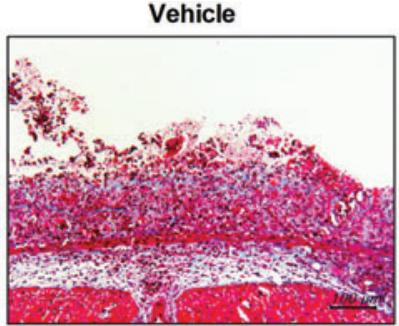

Vehicle

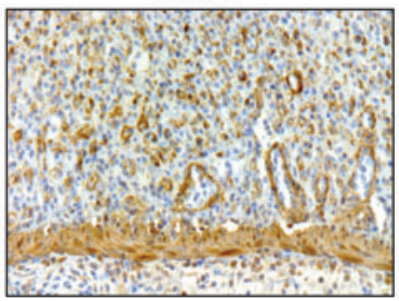

Vehicle

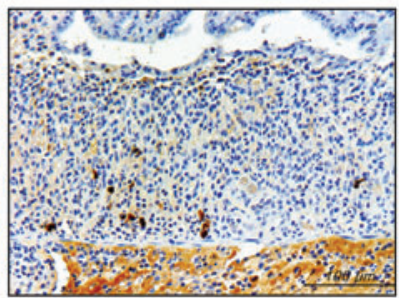

L. acidophilus

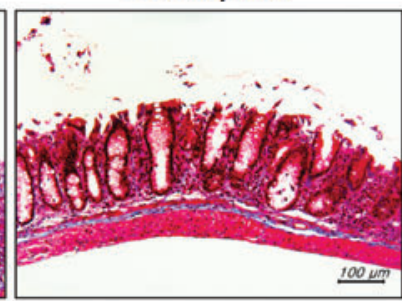

L. acidophilus

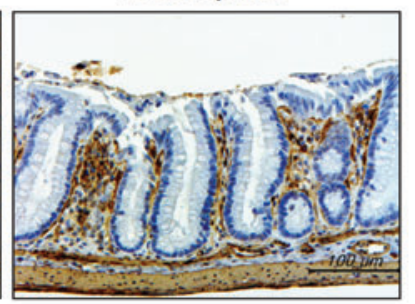

L. acidophilus

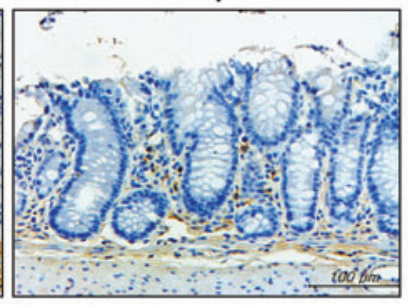

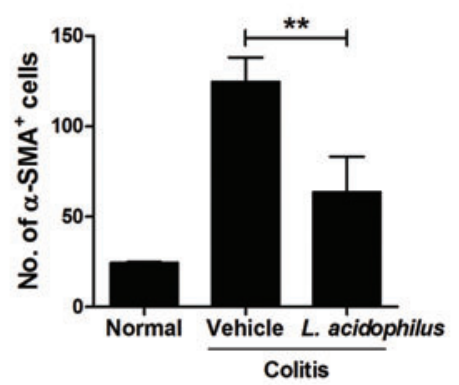

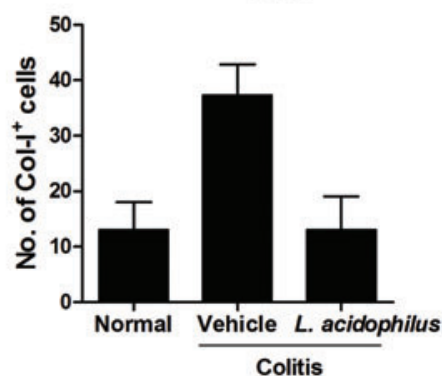

FIG. 5. L. acidophilus reduces colitis-associated intestinal fibrosis. C57BL/6 mice were treated orally with $3 \%$ DSS in distilled water ad libitum from day 0 to 5 . Thereafter, mice received regular drinking water. On the third day after DSS administration, L. acidophilus $\left(8 \times 10^{10} \mathrm{CFU} / \mathrm{kg}\right)$ was administered orally ( $n=3$ /group). On the 10th day after DSS administration, tissue sections were obtained from the colon, and (A) Masson's trichrome staining was performed. The pictures shown depict representative histological features. (B, C) Colon tissue was subjected to immunohistochemical staining for $\alpha$-SMA (b) and Col-I (c). The pictures depict representative histological features, and the results are presented as the mean \pm SD number of antibody-positive cells ( $n=3$ /group). ${ }^{* *} P<.01$ determined using a Student's $t$ test. Vehicle versus $L$. acidophilus treatment. $\alpha$-SMA, $\alpha$-smooth muscle actin. Color images available online at www.liebertpub.com/jmf 
A
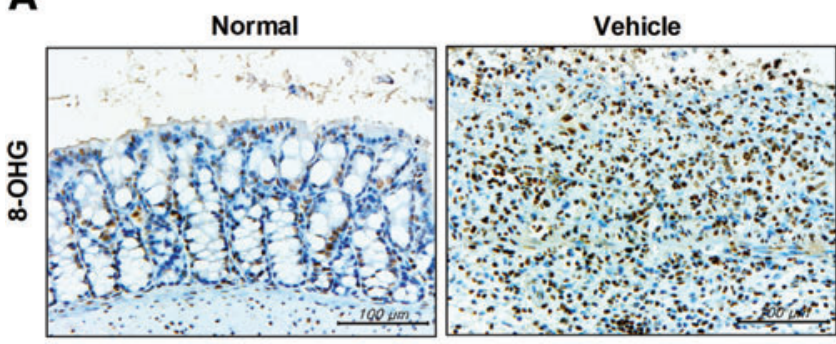

B
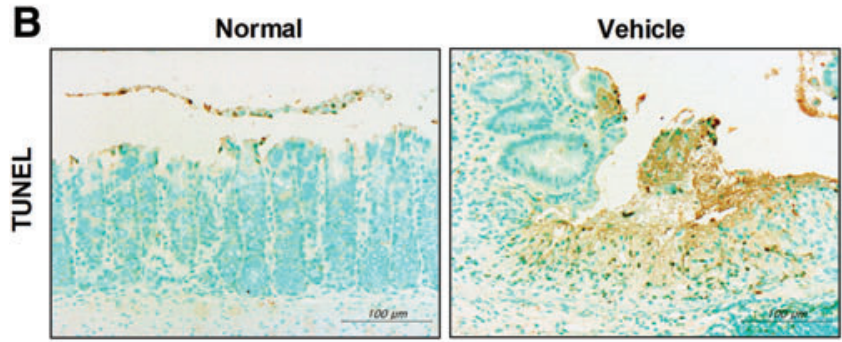
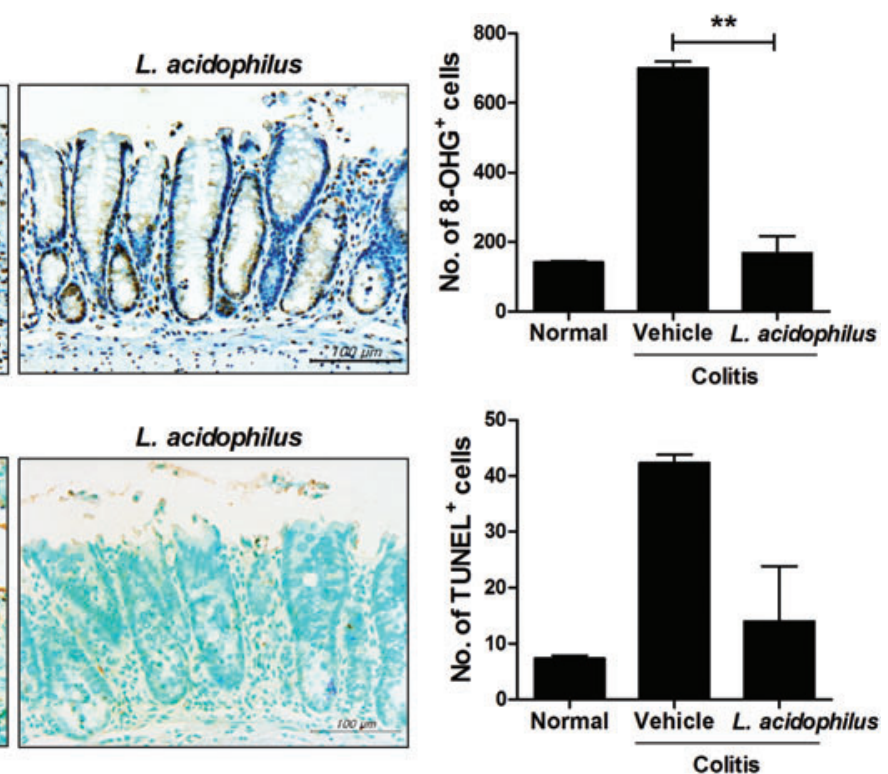

FIG. 6. L. acidophilus reduces oxidative stress and cell apoptosis. C57BL/6 mice were treated orally with $3 \%$ DSS in distilled water ad libitum from day 0 to 5 . Thereafter, mice received regular drinking water. On the third day after DSS administration, L. acidophilus $\left(8 \times 10^{10} \mathrm{CFU} / \mathrm{kg}\right) \mathrm{was}$ administered orally ( $n=3$ /group). On the 10th day after DSS administration, tissue sections were obtained from the colon. (A, B) Colons were subjected to immunohistochemical staining for 8-OHG (A) and TUNEL (B). The pictures depict representative histological features, and the results are presented as the mean \pm SD number of antibody-positive cells $(n=3$ /group). $* * P<.01$ determined using a Student's $t$ test. Vehicle versus $L$. acidophilus-treated group. Color images available online at www.liebertpub.com/jmf

L. acidophilus-treated group compared with the vehicletreated group. Furthermore, L. acidophilus modulated the imbalance between Th17 cell- and Treg cell-related cytokines in this colitis model. Treatment with L acidophilus significantly increased the number of Treg cells and inhibited the production of IL-17, while it increased the production of IL-10 in splenocytes. Moreover, administration of $L$. acidophilus ameliorated collagen deposition in the mucosa of mice with chronic DSS colitis.

The use of probiotics is becoming increasingly important for the prevention and treatment of gastrointestinal conditions. ${ }^{24}$ Probiotics are live microorganisms that, when administered in adequate amounts, exert a health benefit in the host. They play a role in maintaining homeostasis in the gut microbial ecosystem by regulating inflammatory and anti-inflammatory cytokines released by intestinal immunocytes. ${ }^{25-27}$ Currently, classical Lactobacillus and Bifidobacterium species are widely used as health supplements because of their useful roles in health. ${ }^{28}$ Of note, oral administration of L. acidophilus showed therapeutic effects in a DSS-induced colitis model in this study.

It is noteworthy that $L$. acidophilus appeared to regulate the imbalance between Th17 and Treg cells in chronic DSS colitis. There are few studies detailing the effects of $L$. acidophilus in Th17 and Treg cell regulation. In an allergy murine model, L. acidophilus attenuated IL-6 and IL-17 serum levels and downregulated the expression of IL-17A and retinoic acid-related orphan receptor gamma $t$ in the spleen. ${ }^{29}$ Recently, oral administration of L. acidophilus was found to inhibit the expression of IL-17, TNF- $\alpha$, and IL-23, as well as STAT3 and phosphorylated STAT3 in colon tissue in a DSS-induced colitis model. ${ }^{20}$ With respect to Treg cells, treatment with $L$. acidophilus augmented the expansion of Treg cells in intestinal intraepithelial and lamina propria lymphocytes in a 2,4,6-trinitrobenzene sulfonic acid-induced colitis model. ${ }^{30}$ In a murine allergy model, the expression levels of CD25, Foxp3, and TGF- $\beta$ in the spleen were increased in the L. acidophilus-treated group. ${ }^{29}$ Our study showed similar in vivo immunoregulatory effects of L. acidophilus on Th17 and Treg cells in the colon of mice with DSS-induced colitis. Moreover, in vitro treatment of L. acidophilus directly induced the production of Treg cells and IL-10, whereas the production of IL-17 was suppressed in splenocytes. Further studies are needed to further elucidate the relationship between L. acidophilus and Th17/Treg cells.

Notably, we found that L. acidophilus improved DSSinduced intestinal fibrosis. Fibrosis is a chronic inflammationdriven process characterized by the deposition of extracellular matrix components, ultimately leading to organ dysfunction. It is a common and major complication of IBD, which affects more than $30 \%$ of $\mathrm{CD}$ patients and almost $5 \%$ of UC patients. ${ }^{31}$ Despite therapeutic advances in the management of chronic gut inflammation, there have been limited improvements in specific antifibrotic therapies for IBD. In our murine model of DSS-induced chronic colitis and fibrosis, L. acidophilus decreased the levels of $\alpha$-SMA, a marker of activated myofibroblasts, and type I collagen compared with control mice. Recently, IL-17A was implicated in fibrosis of the lungs, liver, and heart. ${ }^{32} \mathrm{IL}-17$ knockout mice also showed 
attenuation of fibrosis in inflammatory skin models, and IL17A promoted cardiac fibroblast proliferation and migration in pulmonary fibrosis models. ${ }^{33,34}$ These studies suggest that IL-17 and Th17 cells are important in promoting fibrosis. Identifying the mechanism underlying the effects of $L$. acidophilus on IL-17-mediated fibrosis would aid the antifibrotic therapy development. In conclusion, we demonstrated in this study that, L. acidophilus can attenuate the symptoms of colitis by regulating Th17 and Treg cells as well as fibrosis. Treatment with L. acidophilus suppressed inflammatory cytokines such as IL-6, TNF- $\alpha$, IL- $\beta$, and IL17. Moreover, in vitro treatment with L. acidophilus directly induced production of Treg cells and IL-10, whereas it suppressed production of IL-17 in splenocytes. L. acidophilus decreased the levels of $\alpha$-SMA, a marker of activated myofibroblasts, and type I collagen compared with the levels in control mice. These results suggest that $L$. acidophilus may be important as a novel therapeutic for IBD by modulating the Th17 and Treg cell balance and fibrosis development.

\section{ACKNOWLEDGMENTS}

This research was supported by a grant from the Korea Health Technology R\&D Project through the Korea Health Industry Development Institute, funded by the Ministry of Health \& Welfare, Republic of Korea (HI15C1062), by a grant from the Korean Health Technology R\&D Project, Ministry for Health \& Welfare, Republic of Korea (HI14C3417), and by a grant from the Korean Health Technology R\&D Project, Ministry for Health \& Welfare, Republic of Korea (HI14C1894).

\section{AUTHOR DISCLOSURE STATEMENT}

No competing financial interests exist.

\section{REFERENCES}

1. Podolsky DK: Inflammatory bowel disease. N Engl J Med 2002; 347:417-429.

2. Xavier RJ, Podolsky DK: Unravelling the pathogenesis of inflammatory bowel disease. Nature 2007;448:427-434.

3. Abraham C, Cho JH: Inflammatory bowel disease. $N$ Engl J Med 2009;361:2066-2078.

4. Van Assche G, Geboes K, Rutgeerts P: Medical therapy for Crohn's disease strictures. Inflamm Bowel Dis 2004;10:55-60.

5. Speca S, Giusti I, Rieder F, Latella G: Cellular and molecular mechanisms of intestinal fibrosis. World J Gastroenterol 2012; 18:3635-3661.

6. Fiocchi C, Lund PK: Themes in fibrosis and gastrointestinal inflammation. Am J Physiol Gastrointest Liver Physiol 2011;300: G677-G683.

7. Galvez J: Role of Th17 Cells in the pathogenesis of human IBD. ISRN Inflamm 2014;2014:928461.

8. Bouma G, Strober W: The immunological and genetic basis of inflammatory bowel disease. Nat Rev Immunol 2003;3:521533.
9. Liu ZJ, Yadav PK, Su JL, Wang JS, Fei K: Potential role of Th17 cells in the pathogenesis of inflammatory bowel disease. World $J$ Gastroenterol 2009;15:5784-5788.

10. Owaga E, Hsieh RH, Mugendi B, Masuku S, Shih CK, Chang JS: Th17 cells as potential probiotic therapeutic targets in inflammatory bowel diseases. Int J Mol Sci 2015;16:20841-20858.

11. Holtta V, Klemetti P, Salo HM, et al.: Interleukin-17 immunity in pediatric Crohn's disease and ulcerative colitis. J Pediatr Gastroenterol Nutr 2013;57:287-292.

12. Ueno A, Jijon $\mathrm{H}$, Chan $\mathrm{R}$, et al.: Increased prevalence of circulating novel IL-17 secreting Foxp3 expressing CD4+ T cells and defective suppressive function of circulating Foxp3+ regulatory cells support plasticity between Th17 and regulatory T cells in inflammatory bowel disease patients. Inflamm Bowel Dis 2013; 19:2522-2534.

13. Sahin A, Calhan T, Cengiz M, et al.: Serum interleukin 17 levels in patients with Crohn's disease: Real life data. Dis Markers 2014;2014:690853.

14. Ito R, Kita M, Shin-Ya M, et al.: Involvement of IL-17A in the pathogenesis of DSS-induced colitis in mice. Biochem Biophys Res Commun 2008;377:12-16.

15. Zhang Z, Zheng M, Bindas J, Schwarzenberger P, Kolls JK: Critical role of IL-17 receptor signaling in acute TNBS-induced colitis. Inflamm Bowel Dis 2006;12:382-388.

16. Maul J, Loddenkemper C, Mundt $\mathrm{P}$, et al.: Peripheral and intestinal regulatory CD4+ CD25(high) $\mathrm{T}$ cells in inflammatory bowel disease. Gastroenterology 2005;128:1868-1878.

17. Gomes-Santos AC, Moreira TG, Castro-Junior AB, et al.: New insights into the immunological changes in IL-10-deficient mice during the course of spontaneous inflammation in the gut mucosa. Clin Dev Immunol 2012;2012:560817.

18. Shibolet O, Karmeli F, Eliakim R, et al.: Variable response to probiotics in two models of experimental colitis in rats. Inflamm Bowel Dis 2002;8:399-406.

19. Guarner F, Malagelada JR: Gut flora in health and disease. Lancet 2003;361:512-519.

20. Chen L, Zou Y, Peng J, et al:: Lactobacillus acidophilus suppresses colitis-associated activation of the IL-23/Th17 axis. J Immunol Res 2015;2015:909514.

21. Suzuki K, Sun X, Nagata M, et al.: Analysis of intestinal fibrosis in chronic colitis in mice induced by dextran sulfate sodium. Pathol Int 2011;61:228-238.

22. Hamamoto N, Maemura K, Hirata I, Murano M, Sasaki S, Katsu $\mathrm{K}$ : Inhibition of dextran sulphate sodium (DSS)-induced colitis in mice by intracolonically administered antibodies against adhesion molecules (endothelial leucocyte adhesion molecule-1 (ELAM-1) or intercellular adhesion molecule-1 (ICAM-1)). Clin Exp Immunol 1999;117:462-468.

23. Zhang L, Zhao Y: The regulation of Foxp3 expression in regulatory $\mathrm{CD} 4(+\mathrm{CD} 25(+) \mathrm{T}$ cells: Multiple pathways on the road. J Cell Physiol 2007;211:590-597.

24. Sullivan A, Nord CE: Probiotics and gastrointestinal diseases. J Intern Med 2005;257:78-92.

25. Erickson KL, Hubbard NE: Probiotic immunomodulation in health and disease. J Nutr 2000;130:403S-409S.

26. Macfarlane GT, Cummings JH: Probiotics, infection and immunity. Curr Opin Infect Dis 2002;15:501-506.

27. Parvez S, Malik KA, Ah Kang S, Kim HY: Probiotics and their fermented food products are beneficial for health. J Appl Microbiol 2006;100:1171-1185. 
28. Stanton C, Gardiner G, Meehan H, et al.: Market potential for probiotics. Am J Clin Nutr 2001;73:476S-483S.

29. Li AL, Meng XC, Duan CC, Huo GC, Zheng QL, Li D: Suppressive effects of oral administration of heat-killed Lactobacillus acidophilus on T helper-17 immune responses in a bovine beta-lactoglobulin-sensitized mice model. Biol Pharm Bull 2013; 36:202-207.

30. Roselli M, Finamore A, Nuccitelli S, et al.: Prevention of TNBSinduced colitis by different Lactobacillus and Bifidobacterium strains is associated with an expansion of gammadeltaT and regulatory $\mathrm{T}$ cells of intestinal intraepithelial lymphocytes. Inflamm Bowel Dis 2009;15:1526-1536.

31. Fichera A, Lovadina S, Rubin M, Cimino F, Hurst RD, Michelassi F: Patterns and operative treatment of recurrent Crohn's disease: A prospective longitudinal study. Surgery 2006;140: 649-654.

32. Liu Y, Zhu H, Su Z, et al.: IL-17 contributes to cardiac fibrosis following experimental autoimmune myocarditis by a PKCbeta/ Erk1/2/NF-kappaB-dependent signaling pathway. Int Immunol 2012;24:605-612.

33. Okamoto Y, Hasegawa M, Matsushita T, et al:: Potential roles of interleukin-17A in the development of skin fibrosis in mice. Arthritis Rheum 2012;64:3726-3735.

34. Valente AJ, Yoshida T, Gardner JD, Somanna N, Delafontaine P, Chandrasekar B: Interleukin-17A stimulates cardiac fibroblast proliferation and migration via negative regulation of the dual-specificity phosphatase MKP-1/DUSP-1. Cell Signal 2012; 24:560-568. 